\title{
Comparison Criteria for Nonlinear Functional Dynamic Equations of Higher Order
}

\author{
Taher S. Hassan ${ }^{1,2}$ and Said R. Grace ${ }^{3}$ \\ ${ }^{1}$ Department of Mathematics, Faculty of Science, University of Hail, Hail 2440, Saudi Arabia \\ ${ }^{2}$ Department of Mathematics, Faculty of Science, Mansoura University, Mansoura 35516, Egypt \\ ${ }^{3}$ Department of Engineering Mathematics, Faculty of Engineering, Cairo University, Orman, Giza 12221, Egypt
}

Correspondence should be addressed to Taher S. Hassan; tshassan@mans.edu.eg

Received 9 February 2016; Accepted 22 May 2016

Academic Editor: Yuriy V. Rogovchenko

Copyright (C) 2016 T. S. Hassan and S. R. Grace. This is an open access article distributed under the Creative Commons Attribution License, which permits unrestricted use, distribution, and reproduction in any medium, provided the original work is properly cited.

We will consider the higher order functional dynamic equations with mixed nonlinearities of the form $x^{[n]}(t)+$ $\sum_{j=0}^{N} p_{j}(t) \phi_{\gamma_{j}}\left(x\left(\varphi_{j}(t)\right)\right)=0$, on an above-unbounded time scale $\mathbb{T}$, where $n \geq 2, x^{[i]}(t):=r_{i}(t) \phi_{\alpha_{i}}\left[\left(x^{[i-1]}\right)^{\Delta}(t)\right], i=1, \ldots, n-$ 1 , with $x^{[0]}=x, \phi_{\beta}(u):=|u|^{\beta} \operatorname{sgn} u$, and $\alpha[i, j]:=\alpha_{i} \cdots \alpha_{j}$. The function $\varphi_{i}: \mathbb{T} \rightarrow \mathbb{T}$ is a rd-continuous function such that $\lim _{t \rightarrow \infty} \varphi_{i}(t)=\infty$ for $j=0,1, \ldots, N$. The results extend and improve some known results in the literature on higher order nonlinear dynamic equations.

\section{Introduction}

In this paper, we consider comparison criteria for higher order nonlinear dynamic equation with mixed nonlinearities of the form

$$
x^{[n]}(t)+\sum_{j=0}^{N} p_{j}(t) \phi_{\gamma_{j}}\left(x\left(\varphi_{j}(t)\right)\right)=0
$$

on an above-unbounded time scale $\mathbb{T}$, where

(i) $n \geq 2$ is an integer, and $x^{[i]}(t):=r_{i}(t) \phi_{\alpha_{i}}\left[\left(x^{[i-1]}\right)^{\Delta}(t)\right]$, $i=1,2, \ldots, n-1, t \in \mathbb{T}$, with $r_{n}=1, \alpha_{n}=1$, and $x^{[0]}=x$;

(ii) $\phi_{\beta}(u):=|u|^{\beta} \operatorname{sgn} u$ for $\beta>0$.

Without loss of generality we assume $t_{0} \in \mathbb{T}$. For $A \subset \mathbb{T}$ and $B \subset \mathbb{R}$, we denote by $C_{\mathrm{rd}}(A, B)$ the space of rightdense continuous functions from $A$ to $B$ and by $C_{\mathrm{rd}}^{1}(A, B)$ the set of functions in $C_{\mathrm{rd}}(A, B)$ with right-dense continuous
$\Delta$-derivatives. Throughout this paper we make the following assumptions:

(iii) $\alpha_{i}, \gamma_{j}>0, i=1,2, \ldots, n-1$ and $j=0,1, \ldots, N$, are constants and $r_{i} \in C_{\mathrm{rd}}\left(\left[t_{0}, \infty\right)_{\mathbb{T}},(0, \infty)\right)$ for $i=$ $1,2, \ldots, n-1$, such that

$$
\begin{aligned}
\int_{t_{0}}^{\infty} r_{i}^{-1 / \alpha_{i}}(s) \Delta s=\infty, & i=1,2, \ldots, n-1 ; \\
\gamma_{j}>\gamma_{0}, & j=1,2, \ldots, l ; \\
\gamma_{j}<\gamma_{0}, & j=l+1, l+2, \ldots, N .
\end{aligned}
$$

(iv) $p_{j} \in C_{\mathrm{rd}}\left(\left[t_{0}, \infty\right)_{\mathbb{T}}, \mathbb{R}^{+}\right)$such that $p_{j} \not \equiv 0, j=0,1, \ldots$, $N$ on $\left[t_{0}, \infty\right)_{\mathbb{T}}$.

(v) $\varphi_{j} \in C_{\mathrm{rd}}(\mathbb{T}, \mathbb{T})$ rd-continuous function such that $\lim _{t \rightarrow \infty} \varphi_{j}(t)=\infty, j=0,1, \ldots, N$, and we let $\varphi(t):=\inf \left\{\varphi_{0}(t), \varphi_{1}(t), \ldots, \varphi_{N}(t)\right\}$ be a nondecreasing function on $\left[t_{0}, \infty\right)_{\mathbb{T}}$.

Recall that the knowledge and understanding of time scales and time scale notation are assumed. For an excellent introduction to the calculus on time scales, see [1-3]. By a solution of (1) we mean a nontrivial real-valued function 
$x \in C_{\mathrm{rd}}^{1}\left[T_{x}, \infty\right)_{\mathbb{T}}$ for some $T_{x} \geq t_{0}$ such that $x^{[i]} \in$ $C_{\mathrm{rd}}^{1}\left[T_{x}, \infty\right)_{\mathbb{T}}, i=1,2, \ldots, n-1$, and $x(t)$ satisfies (1) on $\left[T_{x}, \infty\right)_{\mathbb{T}}$, where $C_{\mathrm{rd}}$ is the space of right-dense continuous functions. An extendable solution $x$ of (1) is said to be oscillatory if it is neither eventually positive nor eventually negative. Otherwise it is said to be nonoscillatory.

In the last few years, there has been an increasing interest in obtaining sufficient conditions for the oscillation/nonoscillation of solutions of different classes of dynamic equations; we refer the reader to [4-13] and the references cited therein. Special cases of (1) have been studied by many authors. When $\alpha_{i}=r_{i}=N=1$ and $\varphi_{i}(t)=t$ for $i=1,2, \ldots, n, p(t)=q_{0}(t)=0$, and $q_{1}(t) \geq 0$, Grace et al. [14] established some oscillation criteria for higher order nonlinear dynamic equation of the form

$$
x^{\Delta^{n}}(t)+p(t)\left(x^{\sigma}(t)\right)^{\gamma}=0,
$$

where $\gamma$ is the ratio of positive odd integers. In paper by Grace [15], some new criteria for the oscillation of the even order dynamic equation

$$
\left[r(t)\left(x^{\Delta^{n-1}}(t)\right)^{\alpha}\right]^{\Delta}+p(t)\left(x^{\sigma}(t)\right)^{\gamma}=0,
$$

where $\alpha$ and $\gamma$ are the ratios of positive odd integers, were given. Recently, Hassan and Kong [16] obtained asymptotics and oscillation criteria for the $n$ th-order half-linear dynamic equation with deviating argument

$$
\left(x^{[n-1]}\right)^{\Delta}(t)+p(t) \phi_{\alpha[1, n-1]}(x(g(t)))=0,
$$

and Grace and Hassan [17] establish oscillation criteria for more general higher order dynamic equation

$$
x^{[n]}(t)+p(t) \phi_{\gamma}\left(x^{\sigma}(\varphi(t))\right)=0 .
$$

The purpose of this paper is to derive comparison criteria for higher order nonlinear dynamic equation with mixed nonlinearities (1).

\section{Preliminaries}

We will employ the following lemmas. Consider the inequality

$$
\left(r(t) \phi_{\alpha}\left(x^{\Delta}(t)\right)\right)^{\Delta}+Q(t) \phi_{\gamma}(x(\varphi(t))) \leq 0,
$$

where $r$ and $Q$ are positive real-valued, rd-continuous functions on $\mathbb{T}$ and $r$ satisfies condition (2), $\varphi: \mathbb{T} \rightarrow \mathbb{T}$ is a rdcontinuous function and $\varphi(t) \rightarrow \infty$ as $t \rightarrow \infty$, and $\alpha$ and $\gamma$ are positive real numbers.

Now, we present the following lemma.

Lemma 1. If inequality (8) has an eventually positive solution, then the equation

$$
\left(r(t) \phi_{\alpha}\left(x^{\Delta}(t)\right)\right)^{\Delta}+Q(t) \phi_{\gamma}(x(\varphi(t)))=0
$$

has also an eventually positive solution.
Proof. Let $x(t)$ be an eventually positive solution of inequality (8). It is easy to see that $x^{\Delta}>0$ eventually. Let $t_{0}$ be sufficiently large so that $x(t)>0, x(\varphi(t))>0$, and $y(t):=r(t) \phi_{\alpha}\left(x^{\Delta}(t)\right)$ for $t \in\left[t_{0}, \infty\right)_{\mathbb{V}}$. Then, in view of

$$
x(t)=x\left(t_{0}\right)+\int_{t_{0}}^{t} \phi_{\alpha}^{-1}\left(\frac{y(s)}{r(s)}\right) \Delta s,
$$

there is $t_{1} \geq t_{0}$ such that $\varphi(t) \geq t_{0}$, for $t \geq t_{1}$. Inequality (8) becomes

$$
y^{\Delta}(t)+Q(t) \phi_{\gamma}\left(x\left(t_{0}\right)+\int_{t_{0}}^{\varphi(t)} \phi_{\alpha}^{-1}\left(\frac{y(s)}{r(s)}\right) \Delta s\right) \leq 0 .
$$

Integrating (11) from $t$ to $v \geq t \geq t_{1}$ and letting $v \rightarrow \infty$, we have

$$
y(t) \geq G(t, y(t)), \quad \text { for } t \in\left[t_{1}, \infty\right)_{\mathbb{T}},
$$

where

$$
\begin{aligned}
& G(t, y(t)) \\
& \quad:=\int_{t}^{\infty} Q(v) \phi_{\gamma}\left(x\left(t_{0}\right)+\int_{t_{0}}^{\varphi(v)} \phi_{\alpha}^{-1}\left(\frac{y(s)}{r(s)}\right) \Delta s\right) \Delta v .
\end{aligned}
$$

Now, we define a sequence of successive approximations $\left\{w_{j}(t)\right\}$ as follows:

$$
\begin{aligned}
w_{0}(t) & :=y(t), \\
w_{j+1}(t) & :=G\left(t, w_{j}(t)\right), \quad j=0,1,2, \ldots .
\end{aligned}
$$

It is easy to show that

$$
\begin{aligned}
0 & <w_{j}(t) \leq y(t), \\
w_{j+1}(t) & \leq w_{j}(t),
\end{aligned}
$$

$$
j=0,1,2, \ldots
$$

Then, the sequence $\left\{w_{j}(t)\right\}$ is nonincreasing and bounded for each $t \geq t_{1}$. This means that we may define $w(t):=$ $\lim _{j \rightarrow \infty} w_{j}(t) \geq 0$. Since

$$
0 \leq w(t) \leq w_{j}(t) \leq y(t), \quad \forall j \geq 0,
$$

we find that

$$
\int_{t_{1}}^{t} w_{j}(s) \Delta s \leq \int_{t_{1}}^{t} y(s) \Delta s .
$$

By Lebesgue's dominated convergence theorem on time scale, one can easily find

$$
w(t)=G(t, w(t)) .
$$

Therefore

$$
\begin{aligned}
w^{\Delta}(t) & =-Q(t) \phi_{\gamma}\left(x\left(t_{0}\right)+\int_{t_{0}}^{\varphi(t)} \phi_{\alpha}^{-1}\left(\frac{w(s)}{r(s)}\right) \Delta s\right) \\
& =-Q(t) \phi_{\gamma}(m(\varphi(t))),
\end{aligned}
$$


where

$$
m(t):=x\left(t_{0}\right)+\int_{t_{0}}^{t} \phi_{\alpha}^{-1}\left(\frac{w(s)}{r(s)}\right) \Delta s .
$$

Then

$$
\begin{aligned}
m(t) & >0, \\
r(t) \phi_{\alpha}\left(m^{\Delta}(t)\right) & =w(t),
\end{aligned}
$$

for $t \geq t_{1}$.

Equation (19) then gives

$$
\left(r(t) \phi_{\alpha}\left(m^{\Delta}(t)\right)\right)^{\Delta}+Q(t) \phi_{\gamma}(m(\varphi(t)))=0 .
$$

Hence (9) has a positive solution $m(t)$. This completes the proof.

The second one is cited from $[18,19]$.

Lemma 2. Assume that (3) holds. Then there exists an N-tuple $\left(\eta_{1}, \eta_{2}, \ldots, \eta_{N}\right)$ with $\eta_{j}>0$ satisfying

$$
\begin{gathered}
\sum_{j=1}^{N} \gamma_{j} \eta_{j}=\gamma_{0}, \\
\sum_{j=1}^{N} \eta_{j}=1 .
\end{gathered}
$$

The next lemma is cited from [17] and improves the wellknown lemma of Kiguradze.

Lemma 3. Assume that (2) holds. If (1) has an eventually positive solution $x$, then there exists an integer $m \in[0, n]$ with $m+n$ being odd such that

$$
\begin{aligned}
& m \geq 1 \\
& \text { implies } x^{[k]}>0 \quad \text { for } k=0,1, \ldots, m-1,
\end{aligned}
$$

eventually, and

$$
\begin{aligned}
& m \leq n \\
& \text { implies }(-1)^{m+k} x^{[k]}>0 \quad \text { for } k=m, \ldots, n,
\end{aligned}
$$

eventually.

\section{Main Results}

In the following main theorem, we will use the following notations: $\alpha[h, k]:=\alpha_{h} \cdots \alpha_{k}$ for $1 \leq h \leq k \leq n-1$ and $\alpha[h, k]=1$ for $h>k$ and, for any $u, v \in \mathbb{T}$, define $R_{j}(v, u)$,
$P_{j}(t)$, and $\bar{P}_{j}(t), j=0, \ldots, n-1$, by the following recurrence formulas:

$$
\begin{aligned}
& R_{j}(v, u) \\
& := \begin{cases}{\left[\int_{u}^{v} \frac{R_{j-1}(s, u)}{r_{m-j+1}(s)} \Delta s\right]^{1 / \alpha_{m-j+1}},} & j=1, \ldots, m, \\
1, & j=0 ;\end{cases} \\
& P_{j}(t) \\
& := \begin{cases}{\left[\frac{1}{r_{n-j}(t)} \int_{t}^{\infty} P_{j-1}(s) \Delta s\right]^{1 / \alpha_{n-j}},} & j=1, \ldots, n-1, \\
p(t), & j=0 ;\end{cases} \\
& \bar{P}_{j}(t) \\
& := \begin{cases}{\left[\frac{1}{r_{n-j}(t)} \int_{t}^{\infty} \bar{P}_{j-1}(s) \Delta s\right]^{1 / \alpha_{n-j}},} & j=1, \ldots, n-1, \\
\bar{p}(t), & j=0,\end{cases}
\end{aligned}
$$

with $p(t):=p_{0}(t)+\prod_{j=1}^{N}\left[p_{j}(t) / \eta_{j}\right]^{\eta_{j}}$ and $\bar{p}(t):=\sum_{j=0}^{N} p_{j}(t)$, provided the improper integrals involved are convergent.

Theorem 4. Assume that for sufficiently large $T \in\left[t_{0}, \infty\right)_{\mathbb{T}}$ the first-order dynamic equation

$$
\begin{aligned}
& z^{\Delta}(t)+K_{m}(t) \phi_{\gamma_{0} / \alpha[1, n]}(z(\varphi(t)))=0, \\
& \qquad \text { for } \varphi(t) \in[T, \infty)_{\mathbb{T}},
\end{aligned}
$$

is oscillatory, where

$$
K_{m}(t):=P_{n-m-1}(t)\left[R_{m}(\varphi(t), T)\right]^{\gamma_{0} / \alpha[m+1, n]},
$$

for every number $m \in\{1, \ldots, n-1\}$ with $m+n$ being odd. Then

(1) if $n$ is even, every solution of (1) is oscillatory,

(2) if $n$ is odd and

$$
\int_{T}^{\infty} \bar{P}_{n-1}(t) \Delta t=\infty
$$

then every solution of (1) either is oscillatory or tends to zero eventually.

Proof. Assume that (1) has a nonoscillatory solution $x$ on $\left[t_{0}, \infty\right)_{\mathbb{T}}$. Then, without loss of generality, $x(t)>0$ and $x\left(\varphi_{j}(t)\right)>0$, for $t \in\left[t_{0}, \infty\right)_{\mathbb{T}}$ and $j=0,1, \ldots, N$. By Lemma 3, there exists an integer $m, 0 \leq m<n$, with $n+m$ being odd such that (24) and (25) hold for $t \geq T_{1} \in\left[t_{0}, \infty\right)_{\mathbb{T}}$.

(I) When $m \geq 1$, from (1), we get

$$
\begin{aligned}
-x^{[n]}(t) & =\sum_{j=0}^{N} p_{j}(t) \phi_{\gamma_{j}}\left(x\left(\varphi_{j}(t)\right)\right) \\
& \geq \sum_{j=0}^{N} p_{j}(t) \phi_{\gamma_{j}}(x(\varphi(t))) \\
& =\phi_{\gamma_{0}}(x(\varphi(t))) \sum_{j=0}^{N} p_{j}(t)[x(\varphi(t))]^{\gamma_{j}-\gamma_{0}} .
\end{aligned}
$$


From (23), we have

$$
\sum_{j=1}^{N} \gamma_{j} \eta_{j}-\gamma_{0} \sum_{j=1}^{N} \eta_{j}=0
$$

Using the arithmetic-geometric mean inequality (see [20, Page 17]), we have

$$
\sum_{j=1}^{N} \eta_{j} v_{j} \geq \prod_{j=1}^{N} v_{j}^{\eta_{j}}, \quad \text { for any } v_{j} \geq 0, \quad j=1, \ldots, N
$$

Then for $t \geq T_{1}$,

$$
\begin{aligned}
& \sum_{j=0}^{N} p_{j}(t)[x(\varphi(t))]^{\gamma_{j}-\gamma_{0}} \\
& \quad=p_{0}(t)+\sum_{j=1}^{N} \eta_{j} \frac{p_{j}(t)}{\eta_{j}}[x(\varphi(t))]^{\gamma_{j}-\gamma_{0}} \\
& \geq p_{0}(t)+\prod_{j=1}^{N}\left[\frac{p_{j}(t)}{\eta_{j}}\right]^{\eta_{j}}[x(\varphi(t))]^{\eta_{j}\left(\gamma_{j}-\gamma_{0}\right)} \\
& \quad=p_{0}(t)+\prod_{j=1}^{N}\left[\frac{p_{j}(t)}{\eta_{j}}\right]^{\eta_{j}}=p(t) .
\end{aligned}
$$

This together with (30) shows that

$$
-x^{[n]}(t) \geq p(t) \phi_{\gamma_{0}}(x(\varphi(t))), \text { for } t \geq T_{1} \text {. }
$$

Integrating above inequality from $t \geq T_{1}$ to $v \in[t, \infty)_{\mathbb{T}}$ and then using the fact that $x$ is strictly increasing and $\varphi$ is nondecreasing, we get

$$
\begin{gathered}
-x^{[n-1]}(v)+x^{[n-1]}(t) \geq \int_{t}^{v} p(s) \phi_{\gamma_{0}}(x(\varphi(s))) \Delta s \\
\geq \phi_{\gamma_{0}}(x(\varphi(t))) \int_{t}^{v} p(s) \Delta s,
\end{gathered}
$$

and by (25) we see that $x^{[n-1]}(v)>0$. Hence by taking limits as $v \rightarrow \infty$ we have

$$
x^{[n-1]}(t) \geq \phi_{\gamma_{0}}(x(\varphi(t))) \int_{t}^{\infty} p(s) \Delta s
$$

which implies

$$
\begin{aligned}
& {\left[x^{[n-2]}(t)\right]^{\Delta}} \\
& \quad \geq \phi_{\alpha_{n-1}}^{-1}\left[\phi_{\gamma_{0}}(x(\varphi(t)))\right]\left[\frac{1}{r_{n-1}(t)} \int_{t}^{\infty} p(s) \Delta s\right]^{1 / \alpha_{n-1}} \\
& \quad=\phi_{\alpha[n-1, n]}^{-1}\left[\phi_{\gamma_{0}}(x(\varphi(t)))\right] P_{1}(t)
\end{aligned}
$$

Integrating above inequality (37) from $t \geq T_{1}$ to $v \in[t, \infty)_{\mathbb{T}}$ and letting $v \rightarrow \infty$, we get

$$
\begin{aligned}
& -x^{[n-2]}(t) \geq \phi_{\alpha[n-1, n]}^{-1}\left[\phi_{\gamma_{0}}(x(\varphi(t)))\right] \int_{t}^{\infty} P_{1}(s) \Delta s \\
& -\left[x^{[n-3]}(t)\right]^{\Delta} \geq \phi_{\alpha[n-2, n]}^{-1}\left[\phi_{\gamma_{0}}(x(\varphi(t)))\right] \\
& \cdot\left[\frac{1}{r_{n-2}(t)} \int_{t}^{\infty} P_{1}(s) \Delta s\right]^{1 / \alpha_{n-2}} \\
& =\phi_{\alpha[n-2, n]}^{-1}\left[\phi_{\gamma_{0}}(x(\varphi(t)))\right] P_{2}(t) .
\end{aligned}
$$

Continuing this process $(n-m-3)$ times, we find

$$
\begin{array}{r}
-\left[x^{[m]}(t)\right]^{\Delta} \geq \phi_{\alpha[m+1, n]}^{-1}\left[\phi_{\gamma_{0}}(x(\varphi(t)))\right] P_{n-m-1}(t), \\
\text { for } t \geq T_{1} .
\end{array}
$$

Also, from (24) and (25), we get

$$
\begin{aligned}
x^{[m-1]}(t)= & x^{[m-1]}\left(T_{1}\right) \\
& +\int_{T_{1}}^{t} \phi_{\alpha_{m}}^{-1}\left(x^{[m]}(s)\right)\left[\frac{1}{r_{m}(s)}\right]^{1 / \alpha_{m}} \Delta s \\
\geq & \phi_{\alpha_{m}}^{-1}\left(x^{[m]}(t)\right) \int_{T_{1}}^{t}\left[\frac{1}{r_{m}(s)}\right]^{1 / \alpha_{m}} \Delta s \\
= & \phi_{\alpha_{m}}^{-1}\left(x^{[m]}(t)\right) R_{1}\left(t, T_{1}\right) .
\end{aligned}
$$

It follows that

$$
\begin{aligned}
& \left(x^{[m-2]}(t)\right)^{\Delta} \\
& \quad \geq \phi_{\alpha[m-1, m]}^{-1}\left(x^{[m]}(t)\right)\left[\frac{R_{1}\left(t, T_{1}\right)}{r_{m-1}(t)}\right]^{1 / \alpha_{m-1}} .
\end{aligned}
$$

Then for $t \in\left[T_{1}, \infty\right)_{\mathbb{T}}$,

$$
\begin{aligned}
& x^{[m-2]}(t) \geq x^{[m-2]}(t)-x^{[m-2]}\left(T_{1}\right) \\
& \geq \int_{T_{1}}^{t} \phi_{\alpha[m-1, m]}^{-1}\left(x^{[m]}(s)\right)\left[\frac{R_{1}\left(s, T_{1}\right)}{r_{m-1}(s)}\right]^{1 / \alpha_{m-1}} \Delta s \\
& \geq \phi_{\alpha[m-1, m]}^{-1}\left(x^{[m]}(t)\right) \int_{T_{1}}^{t}\left[\frac{R_{1}\left(s, T_{1}\right)}{r_{m-1}(s)}\right]^{1 / \alpha_{m-1}} \Delta s \\
& =\phi_{\alpha[m-1, m]}^{-1}\left(x^{[m]}(t)\right) R_{2}\left(t, T_{1}\right) .
\end{aligned}
$$

Analogously, we have

$$
x(t) \geq \phi_{\alpha[1, m]}^{-1}\left(x^{[m]}(t)\right) R_{m}\left(t, T_{1}\right) .
$$

Then for $\varphi(t) \in\left[T_{1}, \infty\right)_{\mathbb{T}}$

$$
x(\varphi(t)) \geq \phi_{\alpha[1, m]}^{-1}\left(x^{[m]}(\varphi(t))\right) R_{m}\left(\varphi(t), T_{1}\right) .
$$


From (39) and (44), we get

$$
\begin{aligned}
- & {\left[x^{[m]}(t)\right]^{\Delta} \geq P_{n-m-1}(t)\left[R_{m}\left(\varphi(t), T_{1}\right)\right]^{\gamma_{0} / \alpha[m+1, n]} } \\
\cdot & \phi_{\gamma_{0} / \alpha[1, n]}\left(x^{[m]}(\varphi(t))\right)=K_{m}(t) \\
\cdot & \phi_{\gamma_{0} / \alpha[1, n]}\left(x^{[m]}(\varphi(t))\right) .
\end{aligned}
$$

Let $z(t):=x^{[m]}(t)>0$; we get

$$
-z^{\Delta}(t) \geq K_{m}(t) \phi_{\gamma_{0} / \alpha[1, n]}(z(\varphi(t)))
$$

or

$$
z^{\Delta}(t)+K_{m}(t) \phi_{\gamma_{0} / \alpha[1, n]}(z(\varphi(t))) \leq 0 .
$$

In view of Corollary 2.3.5 in [21], there exists a positive solution of (27) which contradicts the assumption of the theorem.

(II) When $m=0$ (in this case $n$ is odd), therefore

$$
(-1)^{k} x^{[k]}>0 \text { for } k=0, \ldots, n .
$$

Since $x^{\Delta}<0$ eventually, then $\lim _{t \rightarrow \infty} x(t)=l \geq 0$. Assume that $l>0$. Then for sufficiently large $T_{2} \in\left[T_{1}, \infty\right)_{\mathbb{T}}$, we have $x\left(\varphi_{j}(t)\right) \geq l$ for $t \geq T_{2}$ and $j=0,1, \ldots, N$. It follows that

$$
\phi_{\gamma_{j}}\left(x\left(\varphi_{j}(t)\right)\right) \geq l^{\gamma_{j}} \geq L \quad \text { for } t \in\left[T_{2}, \infty\right)_{\mathbb{T}},
$$

where $L:=\inf _{0 \leq j \leq N}\left\{l^{\gamma_{j}}\right\}>0$. Then from (1), we have

$$
\begin{aligned}
-x^{[n]}(t) & =\sum_{j=0}^{N} p_{j}(t) \phi_{\gamma_{j}}\left(x\left(\varphi_{j}(t)\right)\right) \geq L \sum_{j=0}^{N} p_{j}(t) \\
& =L \bar{p}(t) .
\end{aligned}
$$

Integrating from $t$ to $v \in[t, \infty)_{\mathbb{T}}$, we get

$$
-x^{[n-1]}(v)+x^{[n-1]}(t)=L \int_{t}^{v} \bar{p}(s) \Delta s .
$$

And by $(48)$ we see that $x^{[n-1]}(v)>0$. Hence by taking limits as $v \rightarrow \infty$ we have

$$
x^{[n-1]}(t) \geq L \int_{t}^{\infty} \bar{p}(s) \Delta s
$$

which implies

$$
\begin{aligned}
\left(x^{[n-2]}(t)\right)^{\Delta} & \geq L^{1 / \alpha_{n-1}}\left[\frac{1}{r_{n-1}(t)} \int_{t}^{\infty} \bar{p}(s) \Delta s\right]^{1 / \alpha_{n-1}} \\
& =L^{1 / \alpha_{n-1}} \bar{P}_{1}(t) .
\end{aligned}
$$

Again integrating above inequality from $t$ to $v \in[t, \infty)_{\mathbb{T}}$ and then taking $v \rightarrow \infty$, we get

$$
-x^{[n-2]}(t) \geq L^{1 / \alpha_{n-1}} \int_{t}^{\infty} \bar{P}_{1}(s) \Delta s
$$

which implies

$$
\begin{aligned}
& -\left(x^{[n-3]}(t)\right)^{\Delta} \\
& \geq L^{1 / \alpha[n-2, n-1]}\left[\frac{1}{r_{n-2}(t)} \int_{t}^{\infty} \bar{P}_{1}(s) \Delta s\right]^{1 / \alpha_{n-2}} \\
& =L^{1 / \alpha[n-2, n-1]} \bar{P}_{2}(t) .
\end{aligned}
$$

Continuing this process $(n-3)$ times, we find

$$
-(x(t))^{\Delta} \geq L^{1 / \alpha[1, n-1]} \bar{P}_{n-1}(t) \quad \text { for } t \in\left[T_{2}, \infty\right)_{\mathbb{T}} .
$$

Integrating above inequality $T_{2}$ to $t \in\left[T_{2}, \infty\right)_{\mathbb{T}}$, we get

$$
-x(t)+x\left(T_{2}\right) \geq L^{1 / \alpha[1, n-1]} \int_{T_{2}}^{t} \bar{P}_{n-1}(s) \Delta s .
$$

Hence by (29), we have $\lim _{t \rightarrow \infty} x(t)=-\infty$, which contradicts the fact that $x>0$ eventually. This shows that $\lim _{t \rightarrow \infty} x(t)=$ 0 . This completes the proof.

Theorem 5. Assume that for sufficiently large $T \in\left[t_{0}, \infty\right)_{\mathbb{T}}$ the second-order dynamic equation

$$
\begin{aligned}
& {\left[r_{m}(t) \phi_{\alpha_{m}}\left(z^{\Delta}(t)\right)\right]^{\Delta}+Q_{m}(t) \phi_{\alpha_{m} \gamma_{0} / \alpha[1, n]}(z(\varphi(t)))} \\
& \quad=0, \quad \text { for } \varphi(t) \in(T, \infty)_{\mathbb{T}},
\end{aligned}
$$

is oscillatory, where

$$
Q_{m}(t):=\frac{P_{n-m-1}(t)\left[R_{m}\left(\varphi(t), T_{1}\right)\right]^{\gamma_{0} / \alpha[m+1, n]}}{\left[R_{1}\left(\varphi(t), T_{1}\right)\right]^{\alpha_{m} \gamma_{0} / \alpha[1, n]}},
$$

for every integer number $m \in\{1, \ldots, n-1\}$ with $m+n$ being odd. Then

(1) if $n$ is even, then every solution of (1) is oscillatory,

(2) if $n$ is odd and (29) holds, then every solution of (1) either is oscillatory or tends to zero eventually.

Proof. Assume that (1) has a nonoscillatory solution $x$ on $\left[t_{0}, \infty\right)_{\mathbb{T}}$. Then, without loss of generality, $x(t)>0$ and $x\left(\varphi_{j}(t)\right)>0, j=0,1,2, \ldots, N$ on $\left[t_{0}, \infty\right)_{\mathbb{T}}$. By Lemma 3 , there exists an integer $m, 0 \leq m<n$, with $n+m$ being odd such that (24) and (25) hold for $t \geq T_{1} \in\left[t_{0}, \infty\right)_{\mathbb{T}}$.

(I) When $m \geq 1$, as seen in the proof of Theorem 4, we obtain, for $t \in\left[T_{1}, \infty\right)_{\mathbb{T}}$,

$$
\begin{aligned}
-\left[x^{[m]}(t)\right]^{\Delta} & \geq \phi_{\alpha[m+1, n]}^{-1}\left[\phi_{\gamma_{0}}(x(\varphi(t)))\right] P_{n-m-1}(t), \\
x^{[m-1]}(t) & \geq \phi_{\alpha_{m}}^{-1}\left(x^{[m]}(t)\right) R_{1}\left(t, T_{1}\right) .
\end{aligned}
$$

Hence, we have

$$
\left[\frac{x^{[m-1]}(t)}{R_{1}\left(t, T_{1}\right)}\right]^{\Delta} \leq 0, \quad \text { for } t \in\left(T_{1}, \infty\right)_{\mathbb{T}} .
$$


Since for $t \in\left(T_{1}, \infty\right)_{\mathbb{T}}$,

$$
x^{[m-1]}(t)=\frac{x^{[m-1]}(t)}{R_{1}\left(t, T_{1}\right)} R_{1}\left(t, T_{1}\right),
$$

we have

$$
\begin{aligned}
& \left(x^{[m-2]}(t)\right)^{\Delta} \\
& \quad=\phi_{\alpha_{m-1}}^{-1}\left(\frac{x^{[m-1]}(t)}{R_{1}\left(t, T_{1}\right)}\right)\left[\frac{R_{1}\left(t, T_{1}\right)}{r_{m-1}(t)}\right]^{1 / \alpha_{m-1}} .
\end{aligned}
$$

It follows from (62) that we have, for $t \in\left(T_{1}, \infty\right)_{\mathbb{T}}$,

$$
\begin{aligned}
x^{[m-2]}(t) \geq x^{[m-2]}(t)-x^{[m-2]}\left(T_{1}\right) \\
=\int_{T_{1}}^{t} \phi_{\alpha_{m-1}}^{-1}\left(\frac{x^{[m-1]}(s)}{R_{1}\left(s, T_{1}\right)}\right)\left[\frac{R_{1}\left(s, T_{1}\right)}{r_{m-1}(s)}\right]^{1 / \alpha_{m-1}} \Delta s \\
\geq \phi_{\alpha_{m-1}}^{-1}\left(\frac{x^{[m-1]}(t)}{R_{1}\left(t, T_{1}\right)}\right) \int_{T_{1}}^{t}\left[\frac{R_{1}\left(s, T_{1}\right)}{r_{m-1}(s)}\right]^{1 / \alpha_{m-1}} \Delta s \\
=\phi_{\alpha_{m-1}}^{-1}\left(\frac{x^{[m-1]}(t)}{R_{1}\left(t, T_{1}\right)}\right) R_{2}\left(t, T_{1}\right) .
\end{aligned}
$$

Continuing this process, we have

$$
x(t) \geq \phi_{\alpha[1, m-1]}^{-1}\left(\frac{x^{[m-1]}(t)}{R_{1}\left(t, T_{1}\right)}\right) R_{m}\left(t, T_{1}\right) .
$$

Then for $\varphi(t) \in\left(T_{1}, \infty\right)_{\mathbb{T}}$

$$
x(\varphi(t)) \geq \phi_{\alpha[1, m-1]}^{-1}\left(\frac{x^{[m-1]}(\varphi(t))}{R_{1}\left(\varphi(t), T_{1}\right)}\right) R_{m}\left(\varphi(t), T_{1}\right) .
$$

From (60) and (67), we get

$$
\begin{aligned}
- & {\left[x^{[m]}(t)\right]^{\Delta} \geq \phi_{\alpha_{m} \gamma_{0} / \alpha[1, n]}\left(\frac{x^{[m-1]}(\varphi(t))}{R_{1}\left(\varphi(t), T_{1}\right)}\right) } \\
& \cdot P_{n-m-1}(t)\left[R_{m}\left(\varphi(t), T_{1}\right)\right]^{\gamma_{0} / \alpha[m+1, n]}=Q_{m}(t) \\
& \cdot \phi_{\alpha_{m} \gamma_{0} / \alpha[1, n]}\left(x^{[m-1]}(\varphi(t))\right) .
\end{aligned}
$$

Set $z(t):=x^{[m-1]}(t)>0$; we have, for $t \geq T_{1}$,

$$
\begin{aligned}
- & {\left[r_{m}(t) \phi_{\alpha_{m}}\left(z^{\Delta}(t)\right)\right]^{\Delta} } \\
& \geq Q_{m}(t) \phi_{\alpha_{m} \gamma_{0} / \alpha[1, n]}(z(\varphi(t))),
\end{aligned}
$$

or

$$
\begin{aligned}
& {\left[r_{m}(t) \phi_{\alpha_{m}}\left(z^{\Delta}(t)\right)\right]^{\Delta}+Q_{m}(t) \phi_{\alpha_{m} \gamma_{0} / \alpha[1, n]}(z(\varphi(t)))} \\
& \quad \leq 0 .
\end{aligned}
$$

In view of Lemma 1, there exists a positive solution of (58) which contradicts the assumption of the theorem.

(II) When $m=0$, as shown in the proof of Theorem 4, we show that if (29) holds, then $\lim _{t \rightarrow \infty} x(t)=0$. This completes the proof.
Remark 6. The conclusion of Theorems 4 and 5 remains intact if assumption (29) is replaced by one of the following conditions:

$$
\begin{gathered}
\int_{T}^{\infty} \bar{P}_{0}(t) \Delta t=\infty, \\
\int_{T}^{\infty} \bar{P}_{1}(t) \Delta t=\infty, \\
\vdots \\
\text { or } \int_{T}^{\infty} \bar{P}_{n-2}(t) \Delta t=\infty .
\end{gathered}
$$

Theorem 7. Assume that

$$
\int_{t_{0}}^{\infty} \bar{P}_{2}(t) \Delta t=\infty
$$

And for sufficiently large $T \in\left[t_{0}, \infty\right)_{\mathbb{T}}$, the first-order dynamic equation

$$
\begin{aligned}
& z^{\Delta}(t)+K_{n-1}(t) \phi_{\gamma_{0} / \alpha[1, n]}(z(\varphi(t)))=0, \\
& \qquad \text { for } \varphi(t) \in[T, \infty)_{\mathbb{T}},
\end{aligned}
$$

is oscillatory, where

$$
K_{n-1}(t):=p(t)\left[R_{n-1}(\varphi(t), T)\right]^{\gamma_{0}} .
$$

Then

(1) if $n$ is even, every solution of (1) is oscillatory,

(2) if $n$ is odd, then every solution of (1) either is oscillatory or tends to zero eventually.

Theorem 8. Assume that (72) holds and, for sufficiently large $T \in\left[t_{0}, \infty\right)_{\mathbb{T}}$, the second-order dynamic equation

$$
\begin{aligned}
& {\left[r_{n-1}(t) \phi_{\alpha_{n-1}}\left(z^{\Delta}(t)\right)\right]^{\Delta}} \\
& +Q_{n-1}(t) \phi_{\gamma_{0} / \alpha[1, n-2]}(z(\varphi(t)))=0, \\
& \qquad \text { for } \varphi(t) \in(T, \infty)_{\mathbb{T}},
\end{aligned}
$$

is oscillatory, where

$$
Q_{n-1}(t):=\frac{p(t)\left[R_{n-1}\left(\varphi(t), T_{1}\right)\right]^{\gamma_{0}}}{\left[R_{1}\left(\varphi(t), T_{1}\right)\right]^{\gamma_{0} / \alpha[1, n-2]}} .
$$

Then

(i) if $n$ is even, every solution of (1) is oscillatory,

(ii) if $n$ is odd, every solution of (1) either is oscillatory or tends to zero eventually.

Proof of Theorems 7 and 8. Assume that (1) has a nonoscillatory solution $x$ on $\left[t_{0}, \infty\right)_{\mathbb{T}}$. Then, without loss of generality, it is sufficiently large, such that $x(t)>0$ and $x\left(\varphi_{j}(t)\right)>0$, for $t \in\left[t_{0}, \infty\right)_{\mathbb{T}}$ and $j=0,1, \ldots, N$. By Lemma 3 , there exists 
an integer $m, 0 \leq m<n$, with $n+m$ being odd such that (24) and (25) hold for $t \geq T_{1} \in\left[t_{0}, \infty\right)_{\mathbb{T}}$.

(I) When $m \geq 1$, we claim that (72) implies that $m=n-1$. In fact, if $1 \leq m \leq n-3$, then for $t \geq T_{1}$

$$
\begin{aligned}
x^{[n]}(t) & <0, \\
x^{[n-1]}(t) & >0, \\
x^{[n-2]}(t) & <0, \\
x^{[n-3]}(t) & >0 .
\end{aligned}
$$

Since $x^{\Delta}(t)>0$ on $\left[T_{1}, \infty\right)_{\mathbb{T}}$, then $x(t)>x\left(T_{1}\right):=c_{1}>0$ for $t \geq T_{1}$. Then there exists $T_{2} \in\left[T_{1}, \infty\right)_{\mathbb{T}}$ such that $x\left(\varphi_{j}(t)\right) \geq l$ for $t \geq T_{2}$ and $j=0,1, \ldots, N$. It follows that

$$
\phi_{\gamma_{j}}\left(x\left(\varphi_{j}(t)\right)\right) \geq l^{\gamma_{j}} \geq L, \quad \text { for } t \in\left[T_{2}, \infty\right)_{\mathbb{T}},
$$

where $L:=\inf _{0 \leq j \leq N}\left\{l^{\gamma_{j}}\right\}>0$. Then from (1), we have

$$
\begin{aligned}
-x^{[n]}(t) & =\sum_{j=0}^{N} p_{j}(t) \phi_{\gamma_{j}}\left(x\left(\varphi_{j}(t)\right)\right) \geq L \sum_{j=0}^{N} p_{j}(t) \\
& =L \bar{p}(t) .
\end{aligned}
$$

Integrating from $t$ to $v \in[t, \infty)_{\mathbb{T}}$, we get

$$
-x^{[n-1]}(v)+x^{[n-1]}(t)=L \int_{t}^{v} \bar{p}(s) \Delta s .
$$

And by (48) we see that $x^{[n-1]}(v)>0$. Hence by taking limits as $v \rightarrow \infty$ we have

$$
x^{[n-1]}(t) \geq L \int_{t}^{\infty} \bar{p}(s) \Delta s
$$

which implies

$$
\begin{aligned}
\left(x^{[n-2]}(t)\right)^{\Delta} & \geq L^{1 / \alpha_{n-1}}\left[\frac{1}{r_{n-1}(t)} \int_{t}^{\infty} \bar{p}(s) \Delta s\right]^{1 / \alpha_{n-1}} \\
& =L^{1 / \alpha_{n-1}} \bar{P}_{1}(t)
\end{aligned}
$$

Integrating above inequality from $t$ to $v \in[t, \infty)_{\mathbb{T}}$ and then taking $v \rightarrow \infty$, we get

$$
-x^{[n-2]}(t) \geq L^{1 / \alpha_{n-1}} \int_{t}^{\infty} \bar{P}_{1}(s) \Delta s,
$$

which implies

$$
\begin{aligned}
& -\left(x^{[n-3]}(t)\right)^{\Delta} \\
& \geq L^{1 / \alpha[n-2, n-1]}\left[\frac{1}{r_{n-2}(t)} \int_{t}^{\infty} \bar{P}_{1}(s) \Delta s\right]^{1 / \alpha_{n-2}} \\
& =L^{1 / \alpha[n-2, n-1]} \bar{P}_{2}(t) .
\end{aligned}
$$

Again, integrating above inequality from $T_{2}$ to $t \in\left[T_{2}, \infty\right)_{\mathbb{T}}$ and noting that $x^{[n-3]}>0$ eventually, we get

$$
x^{[n-3]}\left(T_{2}\right)-x^{[n-3]}(t) \geq L^{1 / \alpha[n-2, n-1]} \int_{T_{2}}^{t} \bar{P}_{2}(s) \Delta s .
$$

Then by (72), we have $\lim _{t \rightarrow \infty} x^{[n-3]}(t)=-\infty$, which contradicts the fact that $x^{[n-3]}>0$ on $\left[T_{2}, \infty\right)_{\mathbb{T}}$. This shows that if (72) holds, then $m=n-1$. The rest of proof of (I) is similar to proof (I) of Theorems 4 and 5, respectively, with $m=n-1$ and hence can be omitted.

(II) When $m=0$ (in this case $n$ is odd), therefore

$$
(-1)^{k} x^{[k]}>0 \text { for } k=0, \ldots, n \text {. }
$$

Since $x^{\Delta}<0$ eventually, then $\lim _{t \rightarrow \infty} x(t)=l \geq 0$. Assume that $l>0$. Then for sufficiently large $T_{2} \in\left[T_{1}, \infty\right)_{\mathbb{T}}$, we have $x\left(\varphi_{j}(t)\right) \geq l$ for $t \geq T_{2}$ and $j=1, \ldots, N$. It follows that

$$
\phi_{\gamma_{j}}\left(x\left(\varphi_{j}(t)\right)\right) \geq l^{\gamma_{j}} \geq L \quad \text { for } t \in\left[T_{2}, \infty\right)_{\mathbb{T}},
$$

where $L:=\inf _{0 \leq j \leq N}\left\{l^{\gamma_{j}}\right\}>0$. Then from (1), we have

$$
\begin{aligned}
-x^{[n]}(t) & =\sum_{j=0}^{N} p_{j}(t) \phi_{\gamma_{j}}\left(x\left(\varphi_{j}(t)\right)\right) \geq L \sum_{j=0}^{N} p_{j}(t) \\
& =L \bar{p}(t) .
\end{aligned}
$$

Integrating from $t$ to $v \in[t, \infty)_{\mathbb{T}}$, we get

$$
-x^{[n-1]}(v)+x^{[n-1]}(t)=L \int_{t}^{v} \bar{p}(s) \Delta s .
$$

And using (48) we see that $x^{[n-1]}(v)>0$. Hence by taking limits as $v \rightarrow \infty$ we have

$$
x^{[n-1]}(t) \geq L \int_{t}^{\infty} \bar{p}(s) \Delta s
$$

which implies

$$
\begin{aligned}
\left(x^{[n-2]}(t)\right)^{\Delta} & \geq L^{1 / \alpha_{n-1}}\left[\frac{1}{r_{n-1}(t)} \int_{t}^{\infty} \bar{p}(s) \Delta s\right]^{1 / \alpha_{n-1}} \\
& =L^{1 / \alpha_{n-1}} \bar{P}_{1}(t) .
\end{aligned}
$$

Integrating above inequality from $t$ to $v \in[t, \infty)_{\mathbb{T}}$ and then taking $v \rightarrow \infty$, we get

$$
-x^{[n-2]}(t) \geq L^{1 / \alpha_{n-1}} \int_{t}^{\infty} \bar{P}_{1}(s) \Delta s,
$$

which implies

$$
\begin{aligned}
& -\left(x^{[n-3]}(t)\right)^{\Delta} \\
& \quad \geq L^{1 / \alpha[n-2, n-1]}\left[\frac{1}{r_{n-2}(t)} \int_{t}^{\infty} \bar{P}_{1}(s) \Delta s\right]^{1 / \alpha_{n-2}} \\
& \quad=L^{1 / \alpha[n-2, n-1]} \bar{P}_{2}(t) .
\end{aligned}
$$


Again, integrating above inequality from $T_{2}$ to $t \in\left[T_{2}, \infty\right)_{\mathbb{T}}$ and noting that $x^{[n-3]}>0$ eventually, we get

$$
x^{[n-3]}\left(T_{2}\right)-x^{[n-3]}(t) \geq L^{1 / \alpha[n-2, n-1]} \int_{T_{2}}^{t} \bar{P}_{2}(s) \Delta s .
$$

Then by (72), we have $\lim _{t \rightarrow \infty} x^{[n-3]}(t)=-\infty$, which contradicts the fact that $x^{[n-3]}>0$ on $\left[T_{2}, \infty\right)_{\mathbb{T}}$. This shows that $\lim _{t \rightarrow \infty} x(t)=0$. This completes the proof.

Remark 9. The conclusion of Theorems 7 and 8 remains intact if assumption (72) is replaced by one of the following conditions:

$$
\begin{aligned}
\int_{T}^{\infty} \bar{P}_{0}(t) \Delta t & =\infty \\
\text { or } \int_{T}^{\infty} \bar{P}_{1}(t) \Delta t & =\infty .
\end{aligned}
$$

Theorem 10. Assume that

$$
\int_{T}^{\infty} \bar{P}_{0}(t) \Delta t=\infty
$$

Then

(1) if $n$ is even, every solution of (1) is oscillatory,

(2) if $n$ is odd, then every solution of (1) either is oscillatory or tends to zero eventually.

Proof. Assume that (1) has a nonoscillatory solution $x$ on $\left[t_{0}, \infty\right)_{\mathbb{T}}$. Then, without loss of generality, it is sufficiently large, such that $x(t)>0$ and $x\left(\varphi_{j}(t)\right)>0$, for $t \in\left[t_{0}, \infty\right)_{\mathbb{T}}$ and $j=0,1, \ldots, N$. By Lemma 3 , there exists an integer $m$, $0 \leq m<n$, with $n+m$ being odd such that (24) and (25) hold for $t \geq T_{1} \in\left[t_{0}, \infty\right)_{\mathbb{T}}$.

(I) When $m \geq 1$, this implies that $x(t)$ is strictly increasing on $\left[t_{1}, \infty\right)_{\mathbb{T}}$. Then for sufficiently large $T_{2} \in\left[T_{1}, \infty\right)_{\mathbb{T}}$, we have $x\left(\varphi_{j}(t)\right) \geq l$ for $t \geq T_{2}$. It follows that

$$
\phi_{\gamma_{j}}\left(x\left(\varphi_{j}(t)\right)\right) \geq l^{\gamma_{j}} \geq L \quad \text { for } t \in\left[T_{2}, \infty\right)_{\mathbb{T}},
$$

where $L:=\inf _{0 \leq j \leq N}\left\{l^{\gamma_{j}}\right\}>0$. Equation (1) becomes

$$
\begin{aligned}
-\left(x^{[n-1]}(t)\right)^{\Delta} & =\sum_{j=0}^{N} p_{j}(t) \phi_{\gamma_{j}}\left(x\left(\varphi_{j}(t)\right)\right) \geq L \sum_{j=0}^{N} p_{j}(t) \\
& =L \bar{p}(t) \quad \text { for } t \in\left[t_{2}, \infty\right)_{\mathbb{T}} .
\end{aligned}
$$

Replacing $t$ by $s$ in (98), integrating from $t_{2}$ to $t \in\left[t_{2}, \infty\right)_{\mathbb{T}}$, we obtain

$$
-x^{[n-1]}(t)+x^{[n-1]}\left(t_{2}\right) \geq L \int_{t_{2}}^{t} \bar{p}(s) \Delta s .
$$

Hence by (96), we have $\lim _{t \rightarrow \infty} x^{[n-1]}(t)=-\infty$, which contradicts the fact that $x^{[n-1]}(t)>0$ eventually.

(II) When $m=0$ (in this case $n$ is odd), therefore

$$
(-1)^{k} x^{[k]}>0 \text { for } k=0, \ldots, n .
$$

This implies that $x(t)$ is strictly decreasing on $\left[t_{1}, \infty\right)_{\mathbb{T}}$. Then $\lim _{t \rightarrow \infty} x(t)=l \geq 0$. Assume that $l>0$. Then for sufficiently large $t_{2} \in\left[t_{1}, \infty\right)_{\mathbb{T}}$, we have $x\left(\varphi_{j}(t)\right) \geq l$ for $t \geq t_{2}$. It follows that

$$
\phi_{\gamma_{j}}\left(x\left(\varphi_{j}(t)\right)\right) \geq l^{\gamma_{j}} \geq L \quad \text { for } t \in\left[t_{2}, \infty\right)_{\mathbb{T}},
$$

where $L:=\inf _{0 \leq j \leq N}\left\{l^{\gamma_{j}}\right\}>0$. As is case (I), we get a contradiction with the fact that $x^{[n-1]}(t)>0$ eventually. This shows that $\lim _{t \rightarrow \infty} x(t)=0$. This completes the proof.

\section{Competing Interests}

The authors declare that there are no competing interests regarding the publication of this paper.

\section{References}

[1] M. Bohner and A. Peterson, Dynamic Equations on Time Scales: An Introduction with Applications, Birkhäuser, Boston, Mass, USA, 2001.

[2] M. Bohner and A. Peterson, Eds., Advances in Dynamic Equations on Time Scales, Birkhäuser, Boston, Mass, USA, 2003.

[3] S. Hilger, "Analysis on measure chains-a unified approach to continuous and discrete calculus," Results in Mathematics, vol. 18, no. 1-2, pp. 18-56, 1990.

[4] R. P. Agarwal, M. Bohner, and S. H. Saker, "Oscillation of second order delay dynamic equations," The Canadian Applied Mathematics Quarterly, vol. 13, no. 1, pp. 1-17, 2005.

[5] E. Akin-Bohner and J. Hoffacker, "Oscillation properties of an Emden-Fowler type equation on discrete time scales," Journal of Difference Equations and Applications, vol. 9, no. 6, pp. 603-612, 2003.

[6] M. Bohner and T. S. Hassan, "Oscillation and boundedness of solutions to first and second order forced functional dynamic equations with mixed nonlinearities," Applicable Analysis and Discrete Mathematics, vol. 3, no. 2, pp. 242-252, 2009.

[7] L. Erbe, T. S. Hassan, A. Peterson, and S. H. Saker, "Oscillation criteria for half-linear delay dynamic equations on time scales," Nonlinear Dynamics and Systems Theory, vol. 9, no. 1, pp. 51-68, 2009.

[8] L. Erbe, R. Mert, A. Peterson, and A. Zafer, "Oscillation of even order nonlinear delay dynamic equations on time scales," Czechoslovak Mathematical Journal, vol. 63(138), no. 1, pp. 265279, 2013.

[9] S. R. Grace, R. P. Agarwal, M. Bohner, and D. O’Regan, "Oscillation of second-order strongly superlinear and strongly sublinear dynamic equations," Communications in Nonlinear Science and Numerical Simulation, vol. 14, no. 8, pp. 3463-3471, 2009.

[10] Z. Han, S. Sun, and B. Shi, "Oscillation criteria for a class of second-order Emden-Fowler delay dynamic equations on time scales," Journal of Mathematical Analysis and Applications, vol. 334, no. 2, pp. 847-858, 2007.

[11] T. S. Hassan, "Oscillation criteria for second-order nonlinear dynamic equations," Advances in Difference Equations, vol. 2012, article 171, 2012.

[12] Y. Sun and T. S. Hassan, "Comparison criteria for odd order forced nonlinear functional neutral dynamic equations," Applied Mathematics and Computation, vol. 251, pp. 387-395, 2015. 
[13] T. Sun, W. Yu, and H. Xi, "Oscillatory behavior and comparison for higher order nonlinear dynamic equations on time scales," Journal of Applied Mathematics \& Informatics, vol. 30, no. 1-2, pp. 289-304, 2012.

[14] S. R. Grace, R. P. Agarwal, and A. Zafer, "Oscillation of higher order nonlinear dynamic equations on time scales," Advances in Difference Equations, vol. 2012, article 67, 2012.

[15] S. R. Grace, "On the oscillation of $n$th order dynamic equations on time-scales," Mediterranean Journal of Mathematics, vol. 10, no. 1, pp. 147-156, 2013.

[16] T. S. Hassan and Q. Kong, "Asymptotic and oscillatory behavior of nth-order half-linear dynamic equations," Differential Equations \& Applications, vol. 6, no. 4, pp. 527-549, 2014.

[17] S. R. Grace and T. S. Hassan, "Oscillation criteria for higher order nonlinear dynamic equations," Mathematische Nachrichten, vol. 287, no. 14-15, pp. 1659-1673, 2014.

[18] T. S. Hassan, L. Erbe, and A. Peterson, "Forced oscillation of second order differential equations with mixed nonlinearities," Acta Mathematica Scientia, vol. 31, no. 2, pp. 613-626, 2011.

[19] T. S. Hassan and Q. Kong, "Interval criteria for forced oscillation of differential equations with p-Laplacian, damping, and mixed nonlinearities," Dynamic Systems and Applications, vol. 20, no. 2-3, pp. 279-293, 2011.

[20] E. F. Beckenbach and R. Bellman, Inequalities, Springer, Berlin, Germany, 1961.

[21] R. P. Agarwal, S. R. Grace, and D. O'Regan, Oscillation Theory for Second Order Dynamic Equations, Taylor \& Francis, London, UK, 2003. 


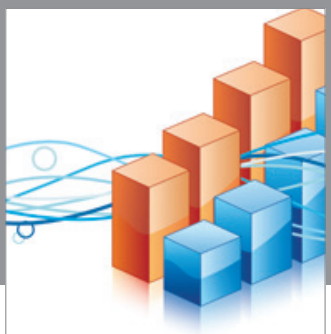

Advances in

Operations Research

vatem alat4

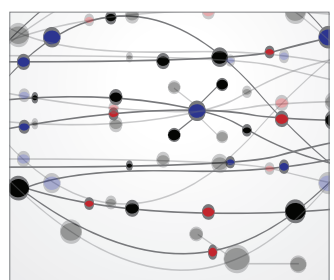

\section{The Scientific} World Journal
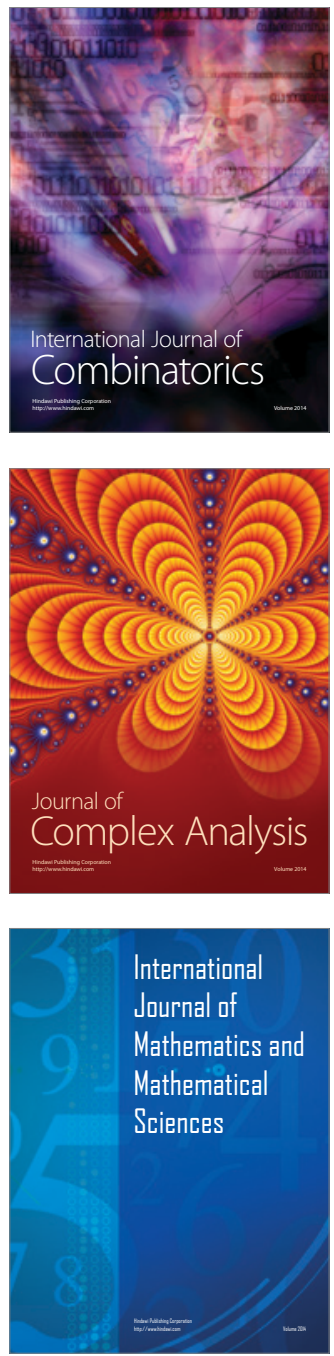
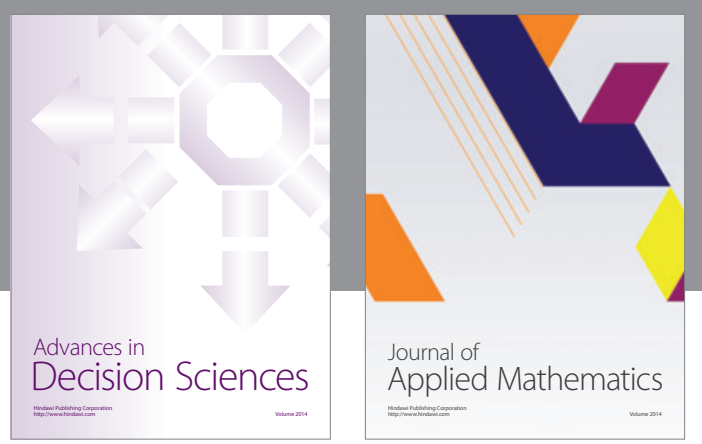

Algebra

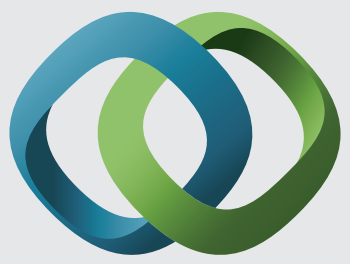

\section{Hindawi}

Submit your manuscripts at

http://www.hindawi.com
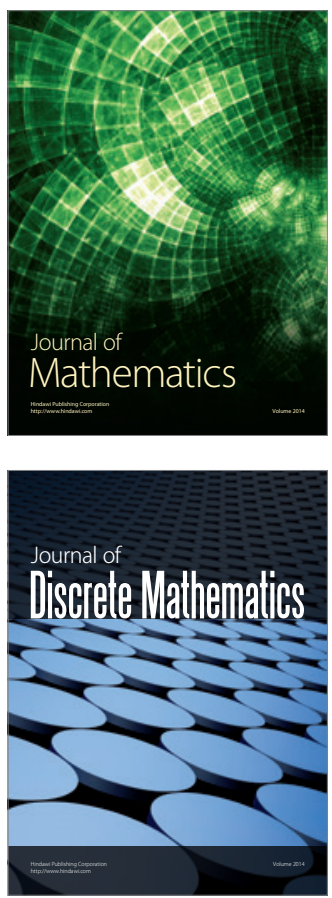

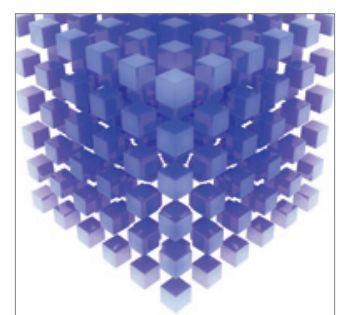

Mathematical Problems in Engineering
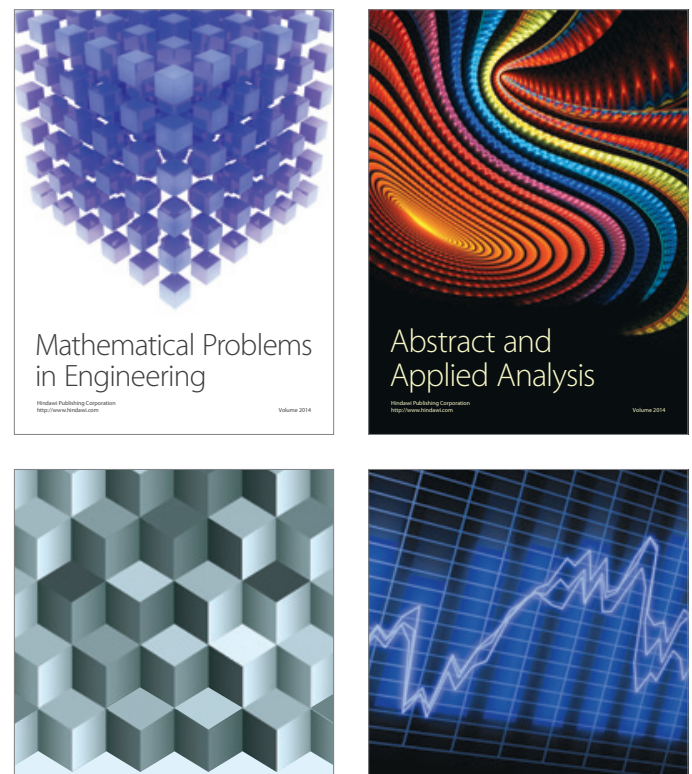

Journal of

Function Spaces

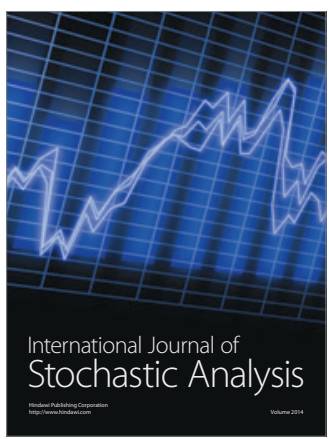

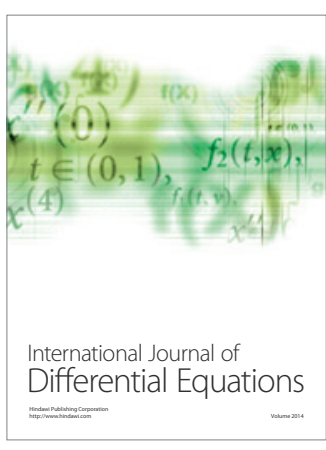
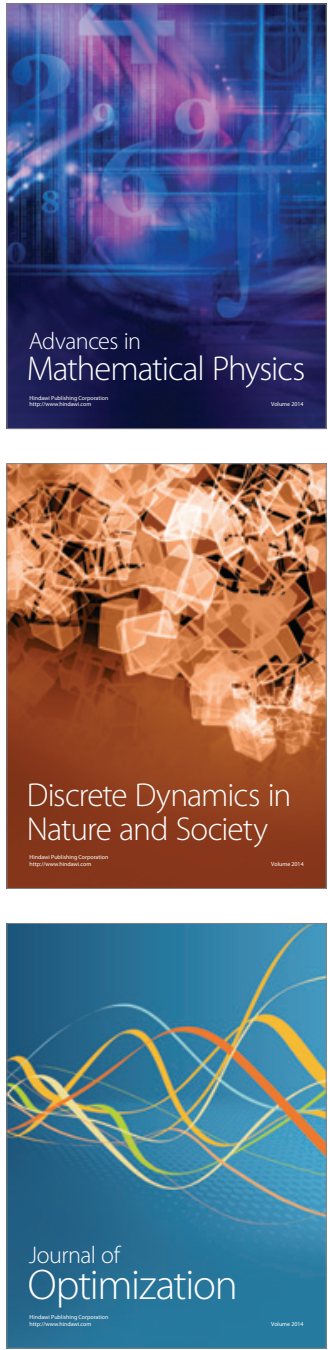